\title{
Symmetric periodic solutions of symmetric Hamiltonians in 1:1 resonance
}

\author{
Yocelyn Pérez ${ }^{1}$ and Claudio Vidal ${ }^{1}$ \\ ${ }^{1}$ Universidad del Bio Bio
}

December 23, 2020

\begin{abstract}
The aim of this work is to prove analytically the existence of symmetric periodic solutions of the family of Hamiltonian systems

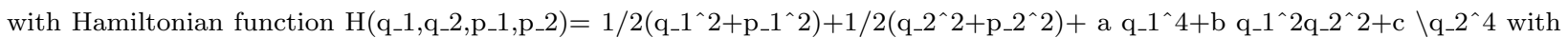
three real parameters $\mathrm{a}, \mathrm{b}$ and $\mathrm{c}$. Moreover, we characterize the stability of these periodic solutions as function of the parameters. Also, we find a first-order analytical approach of these symmetric periodic solutions. We emphasize that these families of periodic solutions are different from those that exist in the literature.
\end{abstract}

\section{Hosted file}

Perez-Vidal.pdf available at https://authorea.com/users/384573/articles/500141-symmetricperiodic-solutions-of-symmetric-hamiltonians-in-1-1-resonance 